\section{INVESTIGACIÓN EN INFORMACIÓN Y COMUNICACIÓN PARA LA CIUDADANÍA: una experiencia educativa de inclusión social}

\author{
Aurora Cuevas-Cerveró
}

RESUMEN: Atender las necesidades básicas de alfabetización de los ciudadanos es una tarea compleja teniendo en cuenta los niveles educativos cada vez más altos requeridos por las sociedades modernas así como la transformación del proceso mismo de alfabetización que hoy ha de incluir las dimensiones relativas a la tecnología, la información y la comunicación. Si trasladamos esta tarea a los sectores más vulnerables de la población nos encontramos ante un gran desafío para crear una metodología de trabajo que permita la integración social de estos sectores. Presentamos resultados de una investigación desarrollada en el nordeste de Brasil que ha tenido como objetivo promover la formación en competencias digitales, informacionales y de comunicación a partir de acciones educativas multidisciplinares dirigida al profesorado y enfocadas a los procesos educativos de la ciudadanía para el acceso a la información atendiendo a tres ejes temáticos: información para salud, información para el acceso a la administración y servicios públicos y acceso a información sobre empleo. Los resultados iniciales indican que las directrices del Curriculum para Profesores sobre Alfabetización Mediática e Informacional de UNESCO resultan especialmente valiosas en los programas pedagógicos de inclusión social. Los trabajos presentados por los profesores participantes tras recibir la formación han resultado de una gran calidad y muestran la necesidad de la formación permanente del profesorado en la búsqueda de la excelencia, especialmente relevante en el trabajo de tipo social. Por otra parte los contenidos presentados fomentan el empoderamiento de la ciudadanía, lo cual implica desarrollar habilidades para defender objetivos y transmitir capacidad de acción.

Palabras clave: Alfabetización mediática e informacional (MIL). Alfabetización informacional. Comunicación. Acceso a la información. Inclusión social.

\footnotetext{
* Doutora em Documentación e Licenciada em Filosofía y Ciencias de la Educación, Espanha. Professora na Universidad Complutense de Madrid, Espanha. Codiretora do Grupo de Investigación "Competencias em informação" da Universidad de Brasilia, Brasil.

E-mail: macuevas@ucm.es.
}

\section{INTRODUCCIÓN}

E $\mathrm{n}$ las últimas décadas el término alfabetización ha incrementado su significado con un gran abanico de matices: alfabetización digital, informacional, audiovisual y multimedia entre otras, convirtiéndose en metáfora de una amplia gama de competencias.
Algunos teóricos hablan de multialfabetización, el concepto fue formulado por el New London Group en la década de los noventa y sostiene que una sociedad multimodal debe cualificar al alumnado ante los múltiples medios y lenguajes de la cultura con un planteamiento integrado de las distintas alfabetizaciones.

Transliteracy es otro de los términos usados, fue acuñado durante las discusiones 
en el seno del Transliteracy Research Group de Montfort University que según Sue Thomas; Chris Joseph, Jess Laccetti y otros (2007) implica la capacidad para leer, escribir e interactuar a través de una amplia gama de plataformas, herramientas y medios de comunicación desde la oralidad y la escritura, pasando por la imprenta, televisión, radio y cine, hasta la tecnología digital de redes sociales.

Pero más allá del debate semántico se revela una aspiración común, aportar a las personas nuevas habilidades que les faciliten la lectura y escritura de un lenguaje cada vez más complejo y les permitan la plena integración social.

Borges y Oliveira (2011) proponen la expresión competencia infocomunicacional enfatizando la convergencia entre competencias informacionales $\mathrm{y}$ en comunicación, tan presente en el momento actual en el que una parte importante de las relaciones sociales es construida a partir de redes y comunidades virtuales.

De acuerdo con la International Federation of Libraries Asociation (IFLA), 2013, entre las principales tendencias de evolución de ambientes informacionales se encuentra la hiperconexión, donde el acceso a la información incluye salud, educación, empleo y participación en las decisiones sociales y políticas, según IFLA las deficiencias en competencias informacionales continúan siendo barreras en este acceso provocando el aumento de la desigualdad social.

Las competencias que los estados deben promover han sido enunciadas por las principales instituciones culturales educativas y bibliotecarias, como UNESCO; IFLA o la Comisión Europea (BAJÓN, 2016) y todas ellas coinciden en la necesidad de fomentar habilidades para acceder, seleccionar, usar, evaluar y comunicar la información para poder transformarla en conocimiento. Este conjunto de habilidades se ha denominado desde los años 70 del pasado siglo information literacy, en español alfabetización en información o informacional. UNESCO publicó en 2011 Alfabetización Mediática $e$ Informacional Curriculum para profesores, transformando la denominación information literacy en Media and Information Literacy (MIL), este documento, pensado como un recurso para profesores, es de gran importancia para lograr los objetivos de la Declaración Grünwald (1982), la Declaración de Alejandría (2005) y la Agenda de Paris de la UNESCO (2007) - relacionadas estrechamente con los objetivos de MIL.

MIL abarca todos los tipos de medios de comunicación y otros proveedores de información como bibliotecas, archivos, museos e Internet, independientemente de las tecnologías utilizadas. La alfabetización mediática e informacional, según consta en el portal de UNESCO

\begin{abstract}
Reconoce el papel fundamental de la información y los medios de comunicación en nuestra vida diaria, son parte central de la libertad de expresión y de información; facultan a los ciudadanos a comprender las funciones de los medios de comunicación y de información, a evaluar críticamente los contenidos y a tomar decisiones fundadas como usuarios y productores de información y contenido mediático.
\end{abstract}

(http://www.unesco.org/new/es/ communication-and-information/mediadevelopment/media-literacy/mil-ascomposite-concept/)

En 2014 UNESCO publica Orientación Normativa y Estratégica de la Alfabetización Mediática e Informacional. A partir de las iniciativas vinculadas a MIL la UNESCO y la Alianza de civilizaciones de las Naciones Unidas (UNAOC) crearon UNESCO UNITWIN UNAOC, red de Universidades para la Alfabetización mediática e informacional y el dialogo multicultural (UAC-MILID). La Cátedra UNESCO-UNAOC MILID UNITWIN surgió tras la firma del acuerdo "Network of Universities on Media and Information Literacy and Intercultural Dialogue" (MILID 2013), suscrito por ocho universidades en Fez, Marruecos, en junio de 2011. En esta publicación se presenta una amplia variedad de proyectos MILID desde la perspectiva de autores provenientes de diversos contextos y países $\mathrm{y}$ ofrece una mirada a diferentes experiencias de Alfabetización mediática que favorecen la implicación de los jóvenes en procesos de producción mediática. El acuerdo se centra en líneas estratégicas como la promoción del desarrollo de la Media and Information Literacy Teachers Training en el mundo, del diálogo intercultural, la integración y el trabajo conjunto entre las universidades que componen la Cátedra 
y las empresas, los medios de comunicación y las organizaciones civiles.

El proyecto de investigación denominado Inclusão cidadã: formação para o acesso à informação $e$ aos serviços públicos, cuyos resultados presentamos, toma como punto de partida el concepto y espíritu de MIL orientándolo a la formación de profesores y vinculándolo a uno de los grandes desafíos de nuestra época, conseguir una democratización plena en el acceso a la información para la ciudadanía, Los otros referentes de nuestro proyecto son la Lei de Acesso à Informação de Brasil y Programa Brasil Sem Miséria, la ley, por definir el acceso a la Información como un Derecho Humano Universal, y el Programa Brasil sin miseria, por incluir a la información como uno de los ejes para la para erradicación de la miseria.

Atender las necesidades básicas de alfabetización de los ciudadanos es una tarea cada vez más compleja, teniendo en cuenta los niveles educativos cada vez más altos requeridos por las sociedades modernas así como la transformación del proceso mismo de alfabetización que hoy ha de incluir las dimensiones relativas a la tecnología, la información y la comunicación. Si trasladamos esta tarea a los sectores más vulnerables de la población nos encontramos ante un gran desafío para crear una metodología de trabajo que pueda servir como base a futuras acciones. En este desafío nos hemos ayudado de las ideas del pedagogo Brasileño Paulo Freire, para este autor la construcción de conocimiento como una práctica colectiva no es una mera transmisión de saberes en una relación verticalizada educadoreducando sino la posibilidad de que juntos, creen y produzcan nuevos sentidos, nuevos conocimientos a partir de la relación establecida, mucha veces no previstos por el educador en el momento de la relación dialógica. Las prácticas educativas, según entiende Freire deben servir para despertar en los sujetos la curiosidad y el deseo por la búsqueda de conocimiento que volcará en el proceso de aprendizaje otras voces indispensables, además de la suya propia. Este despertar de la curiosidad a aprender cosas nuevas no debería ser sometido a los dictámenes de la enseñanza formal, pues, si llega en el diálogo educativo, dará soporte a la formación de su sentido crítico, es decir, a su lectura crítica del mundo (FREIRE, 1983).

\section{METODOLOGÍA}

Se trata de un proyecto de investigación acción, un enfoque de investigación cualitativa que proporciona los medios para llevar a cabo de forma sistemáticas acciones que contribuyan a la resolución de problemas. El método de investigación ha sido el estudio de caso con la intención de que pueda replicarse y funcionar como referencia para futuros proyectos si los resultados resultaran satisfactorios.

El objetivo principal de nuestro proyecto es promover la formación de competencias digitales, informacionales y de comunicación a partir de acciones educativas multidisciplinares enfocadas a los procesos de inclusión social de la ciudadanía para el acceso a la información en comunidades vulnerables atendiendo a tres ejes temáticos: información para salud, información para el acceso a la administración y servicios públicos y acceso a información sobre empleo.

Otro de los objetivos ha sido favorecer la transferencia de conocimiento desde la universidad a los sectores más vulnerables de la sociedad fortaleciendo de esta forma la vocación humanista de la Universidad, aletargada en las últimas décadas en los que se ha favorecido la transferencia a la industria y al mundo empresarial.

Este proyecto además promueve la internacionalización de la ciencia pues ha sido realizado por un equipo interdisciplinar hispano brasileño compuesto por investigadores de la Universidad de Brasilia, Universidad Complutense y Universidad Tiradentes de Aracaju, de las áreas de Ciencias de la Información, Comunicación, Educación y Biblioteconomía y Documentación. Para la parte aplicada de nuestro proyecto hemos contado con la colaboración del Instituto Luciano Barreto Júnior (ILBJ), institución que por sus especiales características ha actuado como centro receptor.

El proyecto se ha pensado con la siguiente estructura:

1. Planteamiento del marco teórico: selección de indicadores que permitan determinar las competencias necesarias para el aprendizaje de la ciudadanía en ambientes tecnológicos: competencias digitales, habilidades informativas y comunicativas para acceso a información y servicios públicos. 
2. Planteamiento del marco aplicativo: creación de un modelo formativo que contemple los principios del curriculum para profesores establecido por MIL y que pueda ser aplicado en contextos informales de formación. Elaboración de los contenidos y diseño de actividades prácticas orientadas a su implementación.

3. Selección de la institución y la muestra para testar el modelo. Tras valorar diferentes opciones se ha determinado contar con el Instituto Luciano Barreto Júnior (ILBJ) por adaptarse a los principios teóricos y éticos que rigen nuestra investigación. Esta Institución, radicada en Aracaju (Brasil) forma parte de la obra social de la Constructora Celi del estado de Sergipe en Brasil y atiende en torno a 1000 alumnos por año, jóvenes en riesgo de exclusión, y cuenta con una plantilla de 20 profesores. Nuestra muestra por tanto reúne a los 20 profesores, 1 asistente social, 1 psicólogo y un pedagogo del ILBJ. La formación redunda en los1000 alumnos de esta institución

4. Aplicación. En una primera fase se realizó la formación del profesorado y demás personal del ILBJ y en una segunda fase los profesores implementaron las acciones formativas diseñadas por ellos mismos a partir de la formación recibida

5. Verificación, análisis y difusión de los resultados.

Toda investigación científica debe cumplir con una serie de requisitos para que pueda ser considerada una investigación ética. Los principios éticos que rigen nuestra investigación siguen las normas éticas internacionales que regulan la investigación con seres humanos, Código de Nuremberg, Declaración Universal de Derechos Humanos y Declaración de Helsinski, tomando en cuenta la especial vulnerabilidad que presentan el colectivo humano con el que trabajamos y garantizando en todo momento la protección de los derechos de los individuos y la validez científica y justificación social del proyecto de investigación. Es de destacar que los investigadores participantes no han interactuado directamente con el colectivo de alumnos sino con los profesores y otros profesionales que atienden a este colectivo en el ILBJ.

\section{PLANTEAMIENTO DEL MARCO TEÓRICO}

Para la selección de los indicadores de competencias se han tenido en cuenta las Recomendaciones de IFLA sobre Alfabetización Informacional y Mediática que aconseja implementar programas de Alfabetización Informacional y Mediática para incrementar las salidas profesionales y las capacidades empresariales de mujeres y grupos marginados, incluyendo emigrantes, desempleados y subempleados (2011) En este documento se entiende la Alfabetización Informacional y Mediática como una suma de conocimientos, habilidades y actitudes relativas a las competencias que deben promoverse en los ciudadanos incluyendo también el pensamiento crítico y las destrezas de comprensión más allá de las fronteras profesionales y educativas. La Alfabetización Informacional y Mediática además debe incluir las fuentes de información multimedia: oral, impresa y digital.

Se han tenido en cuenta también las indicaciones de que UNESCO realiza a través de las directrices sobre MIL. Atendiendo a las indicaciones del Curriculum para Profesores sobre Alfabetización Mediática e Informacional la adquisición de competencias informacionales y de comunicación proporciona a los profesores un conocimiento enriquecido para que favorezcan el empoderaramiento de la ciudadanía (SAORIN; GÓMEZ; HERNÁNDEZ, 2007) y un entendimiento suficiente sobre las funciones de los medios e información en las sociedades democráticas que les permita evaluar el rendimiento de los proveedores de medios e información a la luz de las funciones esperadas. Se espera de esta educación la formación de ciudadanos que potencien el desarrollo de medios y de información independientes y pluralistas.

El modelo de indicadores IDEIAS (CUEVAS-CERVERÓ; GARCÍA-MORENO 2009; CUEVAS-CERVERÓ; SIMEÃO, 2010) presenta el mismo planteamiento y la misma estructura que las recomendaciones propuestas por IFLA y adoptadas por el marco teórico MIL de UNESCO, además de haber sido ya testado en proyectos de inclusión digital orientado a salud, motivo que nos ha llevado a tomarlo como base para la 
definición de indicadores que hemos adoptado en nuestro proyecto.

IDEIAS es un modelo holístico porque integra en un mismo orden las dimensiones digital, informacional y social y además establece expresamente en su diseño el impacto social, la transformación de los colectivos implicados, en este caso un colectivo vulnerable.

Por su condición holística, cada indicador debe estar relacionado en la estructura con todos los de su categoría. El resultado completo incluye 3 grandes tópicos, 18 parámetros y 54 indicadores que, a su vez, están desglosados en competencias que constituyen criterios de valoración.
Este modelo permite intervenir en el diseño mismo de la programación didáctica añadiendo como una parte especialmente valiosa las competencias informacionales, digitales y mediáticas, estrechamente ligadas a las necesidades de la ciudadanía respecto al acceso a la información. Los indicadores nos van a permitir también evaluar la eficacia del proceso educativo una vez concluido, de este modo cumplen una doble función, a partir del modelo se programan los contenidos para más tarde servir en la medición del nivel de competencias obtenidos.

Tabla 1- Indicadores para inclusión digital, informacional y mediática que han servido como base al proyecto Inclusão cidadã: formação para o acesso à informação e aos serviços públicos.

INDICADORES PARA INCLUSIÓN DIGITAL, INFORMACIONAL y MEDIATICA

\begin{tabular}{|c|c|c|}
\hline DESTREZAS & CONOCIMIENTOS & ACTITUDES \\
\hline $\begin{array}{c}\text {-I- } \\
\text { Inclusión digital } \\
\text { Centrado en las competencias } \\
\text { digitales }\end{array}$ & $\begin{array}{c}\text {-II- } \\
\text { Inclusión informacional y mediática } \\
(\text { ALFIN }) \\
\text { Centrado en las competencias } \\
\text { cognitivas y axiológicas }\end{array}$ & $\begin{array}{c}\text {-III- } \\
\text { Inclusión social } \\
\text { Centrado en el aprendizaje } \\
\text { para el desarrollo de la } \\
\text { ciudadanía }\end{array}$ \\
\hline Acceso a las TIC & Acceso a la información & $\begin{array}{c}\text { Autonomía en el } \\
\text { aprendizaje }\end{array}$ \\
\hline $\begin{array}{l}\text { 1. Acceso y distinción del software } \\
\text { ofimático } \\
\text { 2. Manejo de las aplicaciones de } \\
\text { acceso a internet } \\
\text { 3. Manejo de las herramientas } \\
\text { de búsqueda y recuperación de } \\
\text { información }\end{array}$ & $\begin{array}{l}\text { 1. Reconocimiento de la necesidad de } \\
\text { información } \\
2 . \quad \text { Identificación de } \\
\text { fuentes de información } \\
\text { 3. Uso de estrategias para la } \\
\text { localización de información en } \\
\text { diversos medios y fuentes }\end{array}$ & $\begin{array}{l}\text { 1. Consultas en bibliotecas } \\
\text { virtuales } \\
\text { 2. Consultas en recursos } \\
\text { especializados en la red } \\
\text { 3. Lectura de documentos } \\
\text { digitales especializados en red }\end{array}$ \\
\hline $\begin{array}{l}\text { 1. Uso de gestores de contenidos } \\
\text { (blogs, wikis...) } \\
\text { 2. Selección de canales y alertas de } \\
\text { información especializada } \\
\text { 3. Uso de plataformas de enseñanza- } \\
\text { aprendizaje (e-learning) }\end{array}$ & $\begin{array}{l}\text { 1. organización de la información } \\
2 \text {. Integración de nueva } \\
\text { información al acervo personal } \\
\text { 3. aplicar la información incorporada } \\
\text { para la resolución de problemas }\end{array}$ & $\begin{array}{l}\text { Uso efectivo de redes sociales } \\
\text { 1. Frecuencia de consultas } \\
\text { relacionadas con salud en la red. } \\
\text { 2. Variedad en los recursos } \\
\text { utilizados. } \\
\text { 3. Adscripción a colectivos } \\
\text { relacionados con salud en la } \\
\text { red (asociaciones, foros de } \\
\text { discusión, listas de distribución, } \\
\text { redes sociales, etc.) }\end{array}$ \\
\hline
\end{tabular}




\section{Lectura digital}

1. Habilidades para navegar

a través de textos con hipervínculos

2. Lectura en dispositivos electrónicos diversos

3. Uso de sistemas de búsqueda avanzada

\section{Redes digitales}

1. Uso de redes sociales en el ámbito de la salud y empleo

2. Habilidades para el trabajo en colaboración

3. Participación en comunidades virtuales de aprendizaje

\section{Evaluación de las TIC}

1. Evaluación de la facilidad de 1. Evaluación de la búsqueda acceso y conectividad

2. Evaluación de uso de las TIC

3. Actualización y mantenimiento de las TIC

Etica de las TIC

1. uso responsable de las tic

2. Uso de tecnología de código abierto

3. Compartir habilidades tecnológicas con la comunidad
Evaluación de la información

\section{Lectura de la información}

1. Comprensión de la información

2.Lecturaendistintosformatosysoportes

3. Lectura con distintas finalidades (ocio, aprendizaje, trabajo, salud)

\section{Producción y comunicación de la} información

1. Comunicación de la información

2. Cooperación en la producción de conocimiento

3. Generación de nuevo conocimiento (a partir de la información incorporada y aprehendida)

2. Evaluación de la calidad de los contenidos

3. Evaluación de las fuentes de información

\section{Etica de la información}

1. Interés por la información en toda su diversidad (contexto, fuentes y culturas diversas)

2. Respeto de los derechos de libertad y propiedad intelectual 3. Compartir información con la comunidad

\section{Comunicación con} instituciones

1. Conocimiento de la red sanitaria.

2. Conocimiento y uso de documentos institucionales relacionados con salud y empleo (folletos, informes, estudios, etc.).

3. Conocimiento de programas y actividades sanitarias.

\section{Comunicación (social e} intercultural)

1. Elaboración de información sobre salud y empleo (blog, wiki...)

2. Difusión de información sobre salud y empleo.

3. Organización de actividades (charlas,

conferencias, talleres, etc.).

\section{Actitud crítica ante la información}

1. Reconocimiento de la procedencia y calidad de los recursos.

2. Uso de recursos de reconocido prestigio.

\section{Compromiso social}

1. Interés por pertenecer a asociaciones vinculadas a salud y empleo

2. Interés por la participación activa en la red

3. Interés por el activismo en red

Fuente: elaboración propia a partir del modelo IDEIAS Cuevas- Cerveró, Simeão, 2010 
Para la planificación docente se han tenido en cuenta los preceptos siguientes:

1. Se entiende la alfabetización mediática e informacional de forma holística, integrando las diferentes competencias que componen nuestros indicadores (conocimiento, destrezas y actitudes) y se contempla MIL como una herramienta importante para promover el diálogo intercultural y el entendimiento mutuo.

2. Los contenidos curriculares deben hacer posible que los profesores o mediadores faciliten a los estudiantes estas competencias con el propósito de dotarles de las herramientas necesarias para que ellos sean capaces de moverse con la información y los medios de forma autónoma y crítica

3. Se debe preservar el acceso igualitario a la información a todas las personas, con especial atención a mujeres, personas que sufren discapacidad, grupos marginados, pueblos indígenas y minorías étnicas.

\section{APLICACIÓN DEL PROYECTO}

El lugar elegido para la parte aplicada del proyecto fue el Instituto Luciano Barreto júnior (ILBJ), institución sin ánimo de lucro de educación no formal, creada en 2003 y situada en Aracaju, capital del estado de Sergipe, en el Nordeste de Brasil.

ILBJ nace como Responsabilidad Social de empresa constructora Celi. La institución, pensada como instrumento para mejorar la calidad de vida de adolescentes e jóvenes procedentes de familias vulnerables social e económicamente, considera la necesidad de conjugar la producción y democratización del conocimiento con el concepto de educación no formal, favoreciendo la mejoría social e económica a través de la educación (CARVALHO, FREIRE, LIMA, 2015)

Esta elección respondió a dos criterios fundamentales:

1, La misión de ILBJ: Su orientación está dirigida hacia el desarrollo humano, potenciando el mundo del trabajo y el ejercicio de la ciudadanía a través de la infoinclusión social.

2. Sincronía con los objetivos de nuestro proyecto: el ILBJ trabaja en educación no formal con jóvenes y tiene como eje estructurante la adquisición de competencias y habilidades técnicas de informática, estas habilidades sirven de puente entre las diversas acciones socieducativas desarrolladas en la institución.

La formación del profesorado se llevó a cabo de forma semipresencial y utilizando como plataforma de aprendizaje Moodle.

El personal del equipo pedagógico del ILBJ recibió dos cursos presenciales de 20 horas, uno inicial, en mayo de 2014 y otro avanzado en Febrero de 2015. En ambos casos al auxiliarse con la plataforma virtual de aprendizaje se pudo hacer un seguimiento a distancia de los proyectos pedagógicos de los alumnos hasta el momento de su implementación con los jóvenes estudiantes del ILBJ

Tabla 2 - Equipo pedagógico del ILBJ participante en el proyecto

\begin{tabular}{|c|c|c|}
\hline Personal del ILBJ & $\begin{array}{l}\mathrm{N}^{0} \text { Partici- } \\
\text { pantes }\end{array}$ & $\begin{array}{c}\text { Disciplinas } \\
\text { impartidas en el ILBJ }\end{array}$ \\
\hline $\begin{array}{l}\text { E d u c a d o r e s } \\
\text { sociales }\end{array}$ & 19 & $\begin{array}{l}\text { Informática básica } \\
\text { Informática avanzada } \\
\text { Ciudadanía y trabajo } \\
\text { Portugués } \\
\text { Matemáticas } \\
\text { Inglés } \\
\text { Teatro } \\
\text { Comunicación y } \\
\text { medios }\end{array}$ \\
\hline Asistente social & 1 & \\
\hline Psicóloga & 1 & \\
\hline $\begin{array}{l}\text { Asistente de } \\
\text { comunicación }\end{array}$ & 1 & \\
\hline $\begin{array}{l}\text { A s is } t \text { e } n t e \\
\text { pedagógico }\end{array}$ & 2 & \\
\hline
\end{tabular}

Fuente: elaboración propia a partir de los datos aportados por el ILBJ

Los contenidos programáticos del curso se realizaron atendiendo a los indicadores de competencias previamente diseñados. Teniendo en cuenta que todos los alumnos participantes eran titulados superiores el nivel alcanzado y especialmente el nivel de aprehensión de los temas fue excelente como quedó demostrado en las propuestas que ellos mismos materializaron en el propio ILBJ durante el segundo semestre de 2015 
Tabla 3- Ejes temáticos del curso incluyendo la identificación de competencias y objetivos

\begin{tabular}{|c|c|c|}
\hline \multicolumn{2}{|c|}{$\begin{array}{l}\text { COMPETENCIAS EN INFORMACIÓN PARA LA } \\
\text { CIUDADANÍA }\end{array}$} & OBJETIVOS \\
\hline E-EMPLEO & $\begin{array}{l}\text {-Identificación y navegación en la } \\
\text { página del Sistema Nacional de } \\
\text { Empleo } \\
\text {-Conocimientos sobre redacción y } \\
\text { justificación del Curriculum vitae } \\
\text { - Conocimientos sobre bolsas } \\
\text { de trabajo y redes sociales más } \\
\text { destacadas } \\
\text { - Conocimientos sobre solicitudes, } \\
\text { consultas, citas y tramitaciones: } \\
\text { documentos e información } \\
\text { requeridos -Búsqueda y selección de fuentes } \\
\text { de información fiables sobre } \\
\text { empleo }\end{array}$ & $\begin{array}{l}\text { - Conseguir acercar la web del Sistema Nacional de Empleo a los } \\
\text { ciudadanos de forma que sepan identificar la estructura de la } \\
\text { web y sepan a qué tipo de información pueden acceder. } \\
\text { - Capacitar a los alumnos para distinguir qué tipo de } \\
\text { documentación necesitan al solicitar trabajo y cómo se redacta } \\
\text { un Currículum vítae. } \\
\text { - Explicar cómo darse de alta en las diferentes bolsas de trabajo } \\
\text { y redes sociales para la búsqueda d empleo, hacer solicitudes, } \\
\text { realizar citas y otras tramitaciones. Explicar qué información les } \\
\text { pedirán en cada una. } \\
\text { - Explicar a qué tipos de formación profesional pueden acceder } \\
\text { en el mercado de trabajo. } \\
\text { - formar a los alumnos para la búsqueda e identificar fuentes de } \\
\text { información fiables sobre empleo } \\
\text { - Identificar ejemplos de buenas prácticas vinculados a } \\
\text { E-EMPLEO }\end{array}$ \\
\hline E-SALUD & $\begin{array}{l}\text {-Identificación y navegación en la } \\
\text { web de salud de cada comunidad. } \\
\text { - Conocimientos sobre altas, } \\
\text { solicitudes, consultas, citas y } \\
\text { tramitaciones: documentos } \\
\text { e información requeridos y } \\
\text { accesibles } \\
\text {-Búsqueda y selección de fuentes } \\
\text { de información fiables sobre salud }\end{array}$ & $\begin{array}{l}\text {-Acercar la información existente en la web del Ministerio de } \\
\text { Salud a los ciudadanos. } \\
\text { - Conseguir que el alumno sepa identificar la estructura de la web } \\
\text { y sepa navegar por sus diferentes opciones } \\
\text {-Capacitar a los alumnos para saber hacer solicitudes, realizar } \\
\text { citas y otras tramitaciones y que documentación necesitan. } \\
\text {-Lograr que los alumnos conozcan las fuentes de información } \\
\text { fiables sobre salud. } \\
\text {-Identificar ejemplos de buenas prácticas vinculados a E-SALUD }\end{array}$ \\
\hline E-ADMINITRACIÓN & $\begin{array}{l}\text { - Conocimientos sobre el proceso } \\
\text { de tramitación: convocatorias, } \\
\text { solicitud y respuesta y resolución. } \\
\text { - Conocimientos sobre } \\
\text { Normativa y derechos de acceso } \\
\text { a la Administración: privacidad, } \\
\text { acceso a la información y } \\
\text { transparencia } \\
\text {-Documentos oficiales del Estado: } \\
\text { - Conocimientos sobre redes } \\
\text { sociales, correos electrónicos y } \\
\text { privacidad } \\
\text { - Búsqueda y selección de fuentes } \\
\text { de información fiables sobre } \\
\text { información pública }\end{array}$ & $\begin{array}{l}\text { - Explicar de forma general el marco normativo nacional en el } \\
\text { que se encuentra el alumno para saber que normativa le ampara. } \\
\text { - Conseguir que los alumnos analicen y comprendan las leyes de } \\
\text { transparencia y privacidad que les afectan. } \\
\text { - Enseñar a navegar y obtener información pública en los } \\
\text { documentos oficiales del estado } \\
\text { - Enseñar a los alumnos a acceder y solicitar sus expedientes, } \\
\text { títulos y certificados personales, además de enseñar a solicitar } \\
\text { becas relacionadas con su educación y formación } \\
\text { - Cómo funcionan las redes sociales y los correos electrónicos en } \\
\text { cuanto a la salvaguarda de la privacidad. } \\
\text { - Que los alumnos sepan identificar fuentes de información } \\
\text { fiables sobre información pública } \\
\text { - Identificar ejemplos de buenas prácticas vinculados a } \\
\text { E-ADMINISTRACIÓN }\end{array}$ \\
\hline
\end{tabular}

Fuente: elaboración propia 
Los participantes comenzaron a preparar sus propios proyectos pedagógicos para volcarlos en los alumnos del ILBJ en mayo de 2014 y concluyeron en marzo de 2015, fecha en la que comenzaron a aplicar sus proyectos con los alumnos.

El equipo pedagógico del ILBJ decidió aplicar con los alumnos una selección de los proyectos que habían sido diseñados siguiendo las directrices marcadas por la formación recibida. Todos los cursos incluían definición de indicadores, planificación y evaluación.

Los seis cursos seleccionados fueron impartidos durante los meses de julio y agosto de 2015 sobre el total de los alumnos del ILBJ, en torno a 1000 jóvenes con edades comprendidas entre los 14 y 24 años, en grupos de 20 - 30 alumnos en turnos de mañana y tarde y tuvieron una duración de 16 horas.

Proyecto 1. Educación financiera, Creado e impartido por los educadores sociales Bruno Oliveira, Carlos Eduardo Melo, Marcelo Leite e Danielle Santos. Los contenidos del curso han sido incluidos en los módulos de Matemáticas e Informática Básica.

Proyecto 2. Huerta vertical: autocultivo y TIC como herramienta pedagógica, curso creado e impartido por los educadores sociales Edilberto Sousa Rodrigues Filho, Gleide Selma Moraes da Silva Barros, Marisete Cruz, Jonathas Fontes y Thiego Cruz. Los contenidos del curso han sido incluidos en los módulos de Ciudadanía y trabajo, Portugués, actividades interdisciplinares (entre otros conferencias, películas, exposiciones), informática básica,

Proyecto 3. Salud y violencia doméstica. curso creado e impartido por los educadores sociales Ricardo Santana Xisto, Patrícia Santana Santos e Renato Alves dos Anjos. Los contenidos del curso han sido incluidos en los módulos de Informática, inglés, matemáticas y actividades interdisciplinares (entre otros conferencias, películas, exposiciones)

Proyecto 4. Conectándose con el mundo del trabajo: explotación de trabajo infantil, curso creado e impartido por los educadores sociales Daniel David Silva, Keyne Ribeiro, Cleber da Silva e Thiego Cruz. Los contenidos del curso han sido incluidos en los módulos de portugué, informática básica y ciudadanía y trabajo.

Proyecto 5. Educación sexual: tabú en el ámbito familiar, curso creado e impartido por los educadores sociales. Cléber da Silva Conceição; Fábio Mauricio F. Santos; Geane Vieira da Silva; Rita de Cássia Santana. Los contenidos del curso han sido incluidos en los módulos de informática básica y actividades interdisciplinares (entre otros conferencias, películas, exposiciones)

Proyecto 6. Acceso a la información pública, curso creado e impartido por los educadores sociales Ana Paula Machado, Bruno de Oliveira Silva y Marcelo Leite. Los contenidos del curso han sido incluidos en los módulos de matemáticas, informática básica y taller de comunicación y medios.

Estos proyectos fueron presentados por los educadores sociales en el Seminario Inclusão cidadã: formação para o acesso à informação e aos serviços públicos(6). Aunque aún no podemos ofrecer datos para conocer el impacto que estos cursos han tenido sobre el total los alumnos, seguimos trabajando para poder conocer $y$ difundir esta parte de la investigación.

\section{CONCLUSIONES}

La elección de los preceptos de MIL como base teórica de nuestro proyecto y en concreto el Curriculum para Profesores sobre Alfabetización Mediática e Informacional de UNESCO entendemos que ha sido un importante acierto por diversos motivos, entre ellos porque acusa las tendencias actuales que se dirigen hacia la convergencia en una plataforma única por lo que hace un tratamiento holístico que integra las competencias en un solo modelo. Por otra parte está diseñado específicamente para profesores y tiene vocación de integración social lo cual lo hizo especialmente valioso para los objetivos de nuestro proyecto.

El lugar elegido para la implementación del proyecto, Instituto Luciano Barreto Júnior (ILBJ) ha resultado ejemplarizante por su capacidad de escucha y de adoptar críticamente nuestros planteamientos teóricos y aplicativos, mostrando capacidad de cambio y mejora continua y una gran generosidad y entrega hacia su labor social.

Los proyectos pedagógicos presentados por los educadores sociales del ILBJ tras recibir la formación han resultado de una gran calidad y muestran la necesidad de la formación permanente del profesorado en la búsqueda de la 
excelencia, especialmente relevante en el trabajo de tipo social. Por otra parte los contenidos que han presentado fomentan el empoderamiento de la ciudadanía, lo cual implica desarrollar habilidades para defender objetivos y transmitir capacidad de acción. Esa capacidad de acción se traduce en poder y redunda en autonomía y posibilidad de tomar las riendas de la propia vida generando bienestar social y económico.

Nuestro proyecto formativo ha revertido directamente sobre 19 profesores y 1000 alumnos. Consideramos especialmente relevante poder analizar en el futuro el impacto de la formación recibida por los alumnos en su inserción laboral y social, para este empeño continuaremos nuestra vinculación con la investigación colaborativa e internacional de carácter social.

\section{AGRADECIMIENTOS:}

- Conselho Nacional de Desenvolvimento Cien

tífico e Tecnológico (CNPQ). Agencia brasileña financiadora del proyecto de investigación Inclusão cidadã: formação para o acesso à informação e aos serviços públicos, cuyos resultados presentamos. Edital Universal 14/2013 (2013-2015).

- Instituto Luciano Barreto Júnior. Centro receptor que apoyó nuestro proyecto cediéndonos sus instalaciones y personal, aceptando y adaptando nuestros presupuestos teóricos y prácticos.

Artigo recebido em 03/02/20I 7 e aceito para publicação em 28/02/20I7

\section{RESEARCH IN INFORMATION AND COMMUNICATION FOR CITIZENSHIP: an educational experience of social inclusion}

ABSTRACT: $\quad$ Meet the basic needs of literacy of citizens is a complex task when one considers the increasing education levels required by modern societies and the transformation of the literacy process. Literacy today must include the dimensions relating to technology, information and communication. If we transfer this task to the most vulnerable sectors of the population we face a great challenge to create a working methodology that allows social integration. We present results of research conducted in Brazil that has aimed to promote training in digital, informational and communication skills from multidisciplinaryn educational activities aimed at teachers and focused on educational processes of citizenship. This training focuses on access to information according to three themes: health information, information for access to public administration and services and access to employment information. Initial results indicate that the guidelines Literacy Curriculum for Teachers Media and Information (MIL) of UNESCO are particularly valuable in the educational programs of social inclusion. The projects presented by teachers after receiving the training have been of high quality and show the need for teacher training in the pursuit of excellence, especially relevant at work social. Moreover the contents presented promote citizen empowerment, which involves developing skills to defend goals and transmitting capacity for action.

Keywords: $\quad$ Media and Information Literacy (MIL). Information literacy. Communication. Information access. Social inclusión.

\section{REFERENCIAS}

BORGES, J.; OLIVEIRA, L. Competências infocomunicacionais em ambientes digitais. Observatorio (OBS*), v. 5, n. 4, 2011

CARVALHO, D.; FREIRE, V. P.; LIMA, R. D. (2015). Sobre o Instituto Luciano Barreto Junior
Um Olhar Pós Implantação de seu Projeto Institucional Pedagógico. CIAIQ 2015, 2. Avaiable in: <http:// proceedings.ciaiq.org/ index.php/ciaiq2015/article/view/308>. Acces in: 20 dec. 2016

CARLSSON, U.; S. H. CULVER. Media and Information Literacy and Intercultural Dialogue: 
MILID Yearbook 2013. Göteborg, University of Gothenburg, 2013

CUEVAS-CERVERÓ, A.; GARCÍA-MORENO, M.A. Ideias, un modelo de evaluación para inclusión digital y alfabetización informacional orientado a salud. El profesional de la información, vol.9, pg. 240-245, 2009

CUEVAS-CERVERÓ, A.; SIMEÃO, E. (Coord.). Alfabetización informacional e inclusión digital: hacia un modelo de infoinclusión social. Gijón: TREA, 2011

FERNÁNDEZ-BAJÓN, M. T. (2016). Information Policies: Agenda for Digital Inclusion. In: PASSARELLI, B.; STRAUBHAAR, J.; CUEVASCERVERÓ, A. (Eds.). Handbook of Research on Comparative Approaches to the Digital Age Revolution in Europe and the Americas. Hershey, PA: IGI Global, 2016, pp. 275-286

FREIRE, P. Pedagogia do oprimido. Rio de Janeiro: Paz e Terra, 1983.

IFLA . Recomendaciones IFLA sobre Alfabetización Informacional y Mediática. Avaiable in:: <http://www.ifla.org/files/assets/ information-literacy / publications/media-infolit-recommend-es.pdf > Acces in: 20 dec. 2016

IFLA ¿Surcando las olas o atrapados en la marea? Navegando en torno a la evolución de la información 2013. Avaiable in: $<$ http://www. abinia.org/surcando_las_olas_o_atrapados_en_ la_marea.pdf> Acces in: 20 dec. 2016

PASSARELLI, B.; STRAUBHAAR, J.; CUEVASCERVERÓ, A. (Eds.). Handbook of Research on Comparative Approaches to the Digital Age Revolution in Europe and the Americas. Hershey, PA: IGI Global, 2016

SAORIN, T.; GÓMEZ HERNÁNDEZ, J.A. Alfabetizar en tecnologías sociales para la vida diaria y el empoderamiento Anuario Thinkepi, 2014, n. 8, pp. 342-348, 2014

THOMAS, S.; JOSEPH, C; LACCETTI, J. et all. Transliteracy: Crossing divides, 2007. Avaiable in:: http://firstmonday.org/ojs/index.php/fm/ article/view/2060/1908>. Acces in: 20 dec. 2016

UNESCO . Declaration on Media and Information Literacy in the Digital Era. Disponible en: UNESCO (2011) Alfabetización Mediática e Informacional Curriculum para profesores. Avaiable in:: <http://unesdoc.unesco.org/ images/0021/002160/216099S.pdf > Acces in: 20 dec. 2016

UNESCO (2014) Orientación Normativa y Estratégica de la Alfabetización Mediática e Informacional. Avaiable in:: <http://www. unesco.org/new/es/communication-andinformation/resources/news-and-in-focusarticles/all. news/news/unesco_releases_ media_and_information_literacy_policy_and_ strategy_guidelines/\#.VtNIavnhDIU>. Acces in: 20 dec. 2016 